\title{
Model-Based Adaptive Fault Diagnosis in Lithium Ion Batteries: A Comparison of Linear and Nonlinear Approaches
}

\section{Amardeep Sidhu and Afshin Izadian}

Indiana Univ Purdue Univ Indianapolis

\section{Sohel Anwar}

Indiana Univ. Purdue Univ. Indianapolis

\begin{abstract}
In this paper, multiple-model adaptive estimation techniques have been successfully applied to fault detection and identification in lithium-ion batteries. The diagnostic performance of a battery depends greatly on the modeling technique used in representing the system and the associated faults under investigation. Here, both linear and non-linear battery modeling techniques are evaluated and the effects of battery model and noise estimation on the over-charge and over-discharge fault diagnosis performance are studied. Based on the experimental data obtained under the same fault scenarios for a single cell, the non-linear model based detection method is found to perform much better in accurately detecting the faults in real time when compared to those using linear model based method.
\end{abstract}

\section{Introduction}

The developing battery failure modes can be detected and diagnosed accurately using the model based fault diagnosis technique, which falls under the analytical redundancy fault detection and diagnosis paradigm [1]. The model based fault diagnosis performance depends on the accurate representation of the process and fault models while maintaining robustness in the presence of unknown disturbances, model uncertainties, and noise $[\underline{2}, \underline{3}]$. In the presence of these undesirable conditions, the important fault information carrying residuals get corrupted and hence result in loss of accurate fault detection and diagnosis.

State estimation [4] [ㄷ] and specifically the use of Luenberger observer (LO) for fault detection and diagnosis can be found in [6] where the fault detection and diagnosis on a string of Li-ion batteries using a bank of reduced order observers was implemented. LO provides little or no robustness with respect to measurement noise.

The use of Kalman filters under the paradigm of observer based fault diagnosis for fault detection and diagnosis in $\mathrm{Li}$-ion batteries is given in $[\underline{7}, \underline{8}]$, where the optimal filter shows strong robustness to noise and the adaptive nature of the multiple model adaptive estimation (MMAE) algorithm ensures accurate fault detection. MMAE is a robust fault detection and identification technique with extensive applications in the aerospace industry $[\underline{9}, \underline{10}]$ and recently in the fault detection of micro electro mechanical systems $[\underline{11}, \underline{12}]$. With the application of Kalman filters and extended Kalman filters under multiple model adaptive estimation technique $[\underline{12}, \underline{13}, \underline{14}]$, the performance deterioration can be minimized to a very large extent. While the use of observer based fault diagnosis through MMAE offers good li-ion battery diagnosis performance [7], for it to be sensitive to minute battery performance shift, as in the case of condition monitoring, it is imperative that the li-ion battery model offers good fault detection and diagnosis characteristics and the system noise is correctly estimated [11]. By avoiding noise over or under estimation and appropriate model selection the quality of residuals can be enhanced.

In this paper, the linear and non-linear li-ion battery models are constructed using the impedance spectroscopy experimental results. These models are tested for fault detection and diagnosis (FDD) performance in over charge and over discharge failure of the li-ion battery cell. Further, the FDD performance as a function of noise estimation is also studied.

\section{Battery Model}

The li-ion battery is modeled as an equivalent circuit $[\underline{4}][\underline{5}]$ and $[\underline{15}$, 16] as shown in Figure 1.

Applying Kirchhoff's voltage law on the model circuit given in Figure 1, the voltage across the capacitor $\mathrm{C}$ is given by,

$$
\dot{V}_{C}=-\frac{V_{C}}{R C}+\frac{I_{L}}{C}
$$

This is the author's manuscript of the article published in final edited form as: 
where $V_{C}$ is the voltage across the capacitor $C, I_{L}$ is the charge/ load current. According to the sign convention used in this study, the negative sign of $I_{L}$ represents the discharge, while the positive sign indicates the charge. The voltage across the double layer capacitive element $C_{d l}$ is given by,

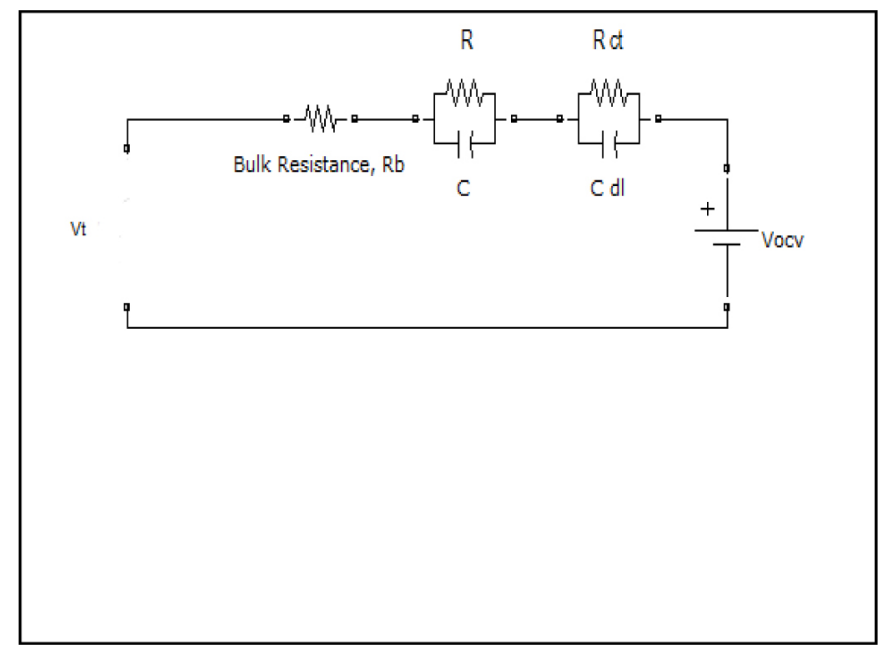

Fig. 1. Li-ion battery equivalent circuit model, $R_{b}$ is the bulk electrolyte resistance; $C, R$, are the capacitance and resistance representing local property of the electrode. $R_{c t}$ is the charge transfer resistance, $C_{d l}$ is the double layer capacitance $[\underline{17}, \underline{18}] . V_{O C V}$ is the open circuit voltage $(\mathrm{OCV})$ of the cell, as a function of the battery state of charge (SOC) [19].

$$
V \dot{c}_{d l}=-\frac{V_{C_{d l}}}{R_{c t} C_{d l}}+\frac{I_{L}}{C_{d l}}
$$

where $V_{C_{d l}}$ is the voltage across $C_{d l}$.

The terminal voltage, $V_{t}$ is given by,

$$
V_{t}=I_{L} R_{b}+V_{C}+V_{C_{d l}}+V_{O C V}
$$

where, $I_{L} R_{b}$ represents voltage drop across the bulk resistance $R_{b}$, and $V_{O C V}$ represents the open circuit voltage of the cell. The SOC is the ratio of the remaining capacity to the nominal capacity of the battery cell and is given by,

$$
\operatorname{SOC}(t)=\operatorname{SOC}(0)+\int_{0}^{t} \frac{\eta I_{L}(\tau)}{C_{n}} d \tau
$$

where $\operatorname{SOC}(0)$ represents the initial state of charge, $C_{n}$ represents the battery cell nominal capacity in Amperehour, and $\eta$ represents the coulomb efficiency given by [ㅈ]:

$$
\eta=\left\{\begin{array}{c}
1, \text { charging } \\
0.98, \text { discharging }
\end{array}\right.
$$

The discrete time counterpart of (4) is given by,

$$
\operatorname{SOC}(k)=\operatorname{SOC}(k-1)+\frac{\eta I_{L}(k-1) \Delta t}{C_{n}}
$$

$$
\begin{gathered}
V_{C}(k)=\left(e^{-\frac{\Delta t}{R C}}\right) V_{C}(k-1)+R\left[1-\left(e^{-\frac{\Delta t}{R C}}\right)\right] I_{L}(k-1), \\
V_{C_{d l}}(k)=\left(e^{-\frac{\Delta t}{R_{c t} C_{d l}}}\right) V_{C_{d l}}(k-1)+R_{c t}[1- \\
\left.\left(e^{-\frac{\Delta t}{R_{c t} C_{d l}}}\right)\right] I_{L}(k-1),
\end{gathered}
$$

where $\Delta t$ is the sample time. The difference equations given above can be represented in the state space form given by,

$$
\begin{gathered}
x[k+1]=G x[k]+H u[k]+\Gamma w[k], \\
y[k]=C x[k]+D u[k]+v[k] .
\end{gathered}
$$

where $x[k]$ is the state vector of the system, and at any sample $k$, is given by $\left[\operatorname{SOC}(k) \quad V_{c}(k) \quad V_{C_{d l}}(k)\right]^{T}$.

$G=\left[\begin{array}{ccc}1 & 0 & 0 \\ 0 & e^{-\frac{\Delta t}{R C}} & 0 \\ 0 & 0 & e^{-\frac{\Delta t}{R_{c t} C_{d l}}}\end{array}\right]$ is the discrete state matrix,

$H=\left[\frac{\eta \Delta t}{C_{n}} \quad R\left[1-\left(e^{-\frac{\Delta t}{R C}}\right)\right] \quad R_{c t}\left[1-\left(e^{-\frac{\Delta t}{R_{c t} C_{d l}}}\right)\right]\right]^{T}$ is the

discrete input vector, $C$ is the output vector, and $D$ is the feed forward term. $\Gamma$ is the models input noise matrix, $w$ is the input noise with zero mean and a variance of

$$
E\left\{w_{n}[l] w_{n}{ }^{T}[m]\right\}=\left\{\begin{array}{ll}
Q, & l=m \\
0, & l \neq m
\end{array},\right.
$$

and $v$ is the measurement noise, independent from $w$, with zero mean value as

$$
E\left\{v_{n}[l] v_{n}^{T}[m]\right\}= \begin{cases}R, & l=m \\ 0, & l \neq m\end{cases}
$$

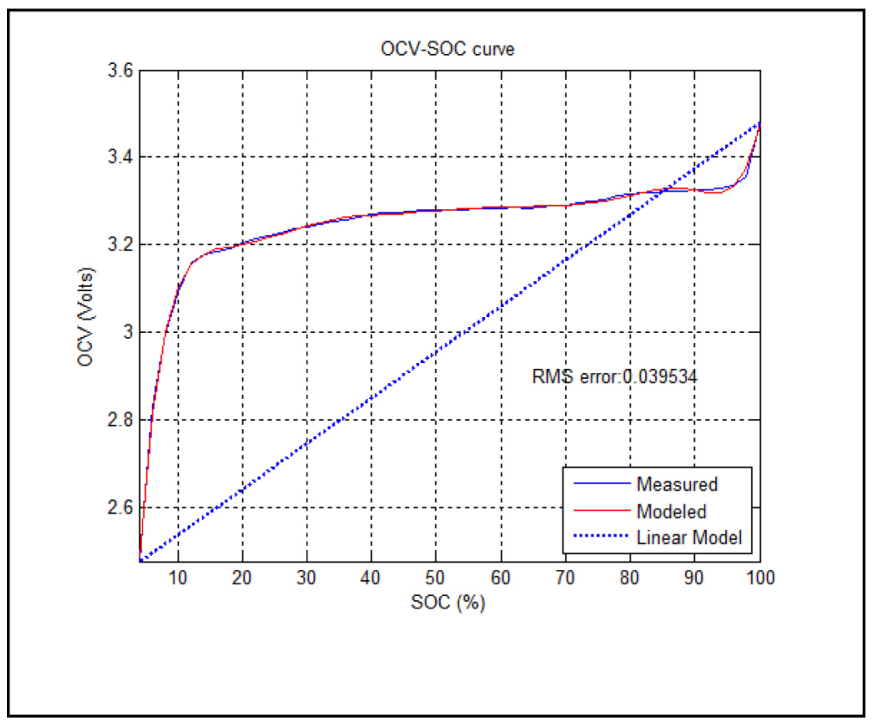

Fig. 2. Experimental OCV-SOC curve for LiFePO4 battery cell. 
In this formulation, $Q$ and $R$ are the process and measurement noise variances, respectively. The process and measurement white Gaussian noise is generated using the polar method [21].

As mentioned earlier, the OCV is a function of SOC and can be found experimentally [22]. For this study the OCV-SOC trend, shown in Figure 2 , is used. The data was recorded from a sample LiFePO4 battery cell operating at room temperature at the Energy Systems and Power Electronics Laboratory (ESPEL) at IUPUI.

\section{Over-Charge and Over-Discharge}

The over-charge failure of battery cells can be attributed to a combination of factors such as excessive temperature along with cell construction and design [23] and can lead to violent thermal runaways. The over-discharge failures are caused due to detrimental copper plating occurring at the negative electrode which can further lead to thermal runaways under severe over-discharge [24]. With over-charge and over-discharge in a li-ion battery, its model parameters show marked variation trend from healthy battery parameters. This kind of variation is expected and is captured excellently using IS on the respective cycled batteries. For example, the bulk resistance, $R_{b}$, shows a more substantial increase under over-charge than in over-discharge; the charge transfer resistance, $R_{c t}$, and constant phase element (CPE) arm resistance, $R$, increase with both failure modes. This increment is more profound under overcharge than in over-discharge. The double layer capacitance, $C_{d l}$, and the capacitor representing the $\mathrm{CPE}, \mathrm{C}$, increase rapidly with overdischarge, while under over-charge they show a relatively smaller change with a gradual decrease.

\section{Linear Model}

Approximating the OCV-SOC behavior by a straight line, as shown by the dotted line in Figure 2, results in a linear battery model. The linear OCV-SOC relationship is given by,

$$
V_{O C V}=m S O C+d,
$$

where $m=\frac{d(\mathrm{OCV})}{d(\mathrm{SOC})}$ is the slope of the line and $d$ is the no-charge OCV. Using this linear relationship results in a linear li-ion battery model where the terminal voltage of (3) can now be given by,

$$
V_{t}=I_{L} R_{b}+V_{C}+V_{C_{d l}}+m S O C+d,
$$

Comparing (11) with the discrete time state space form, we get, $C=$ [ $m$ r 111 ],$D=R_{b}$, and $d$ can be considered as disturbance.

\section{Non-Linear Model}

The OCV-SOC relationship can also be captured by fitting a polynomial to the experimental curve, as shown by modeled curve in Figure 2. Through trial, a ninth degree polynomial gives the best fit to the experimental data with RMS error of 0.039 . This polynomial is given by,

$$
\begin{aligned}
& V_{O C V}=a_{1}\left(S_{O C}{ }^{9}\right)+a_{2}\left(\operatorname{SOC}^{8}\right)+a_{3}\left(\operatorname{SOC}^{7}\right)+a_{4}\left(\operatorname{SOC}^{6}\right) \\
& +a_{5}\left(\operatorname{SOC}^{5}\right)+a_{6}\left(\operatorname{SOC}^{4}\right)+a_{7}\left(\operatorname{SOC}^{3}\right)+a_{8}\left(\operatorname{SOC}^{2}\right) \\
& +a_{9}(S O C)+a_{10} \text {, }
\end{aligned}
$$

where $\alpha_{1}=0.0385, \alpha_{2}=-0.01936, \alpha_{3}=-0.169, \alpha_{4}=0.06142, \alpha_{5}=$ $0.2328, \alpha_{6}=-0.05715, \alpha_{7}=-0.08321, \alpha_{8}=0.0005257, \alpha_{9}=0.03205$, $\alpha_{10}=3.297$.

The resulting non-linear battery model can now be represented in the general form given by,

$$
\begin{gathered}
x[k]=f\left(x[k-1], I_{L}[k-1]\right)+\Gamma w[k] \\
y[k]=h\left(x[k], I_{L}[k]\right)+v[k]
\end{gathered}
$$

where the function $f$ is given by,

$$
\begin{aligned}
& f[k-1] \\
& =\left[\begin{array}{c}
\operatorname{SOC}[k-1]+\frac{\eta \Delta \mathrm{t} I_{L}[k-1]}{C_{n}} \\
\left(e^{-\frac{\Delta t}{R C}}\right) V_{C}[k-1]+R\left[1-\left(e^{-\frac{\Delta t}{R C}}\right)\right] I_{L}[k-1] \\
\left(e^{-\frac{\Delta t}{R_{c t} C_{d l}}}\right) V_{C_{d l}}[k-1]+R_{c t}\left[1-\left(e^{-\frac{\Delta t}{R_{c t} C_{d l}}}\right)\right] I_{L}[k-1]
\end{array}\right]
\end{aligned}
$$

and the function $h$ is given by,

$$
\begin{aligned}
& h(k) \\
& =I_{L}(k) R_{b}+V_{C}+V_{C_{d l}} \\
& +a_{1}\left(S O C^{9}\right)+a_{2}\left(S O C^{8}\right)+a_{3}\left(S O C^{7}\right)+a_{4}\left(S O C^{6}\right) \\
& \quad+a_{5}\left(S O C^{5}\right)+a_{6}\left(S O C^{4}\right)+a_{7}\left(S O C^{3}\right)+a_{8}(S O C \\
& +a_{9}(S O C)+a_{10} .
\end{aligned}
$$

For both the linear and non-linear battery models, the lumped electrical elements and their associated scalar values represent the model of the battery cell at any given time. Considering the different values for the electrical elements $R_{b}, R, C, R_{c t}$, and $C_{d l} ; n$ distinct models can be obtained, each representing a signature fault or the health of the battery cell.

\section{Design of Experiments}

Faults occurring in the li-ion battery result in appreciable change in the battery operation and hence influence the battery model parameter values. Considering different health stages of the battery, several models can be designed to represent the $n$ signature faults of the battery system. In this study we will primarily focus on overcharge and over-discharge failure conditions. The model based fault diagnosis structure is as shown in Figure 3.

As discussed earlier, the healthy and fault model parameters are extracted using IS. The IS technique involves applying a small amplitude frequency sweep to the battery system. The frequency sweep usually rides on top of a load current or charging current to capture the impedance data for over-discharge and over-charge 
respectively. The test subject selected for this study is A123 18650 $\mathrm{LiFePO}_{4}$ battery cell [25]. Tables I and II give the impedance spectroscopy results for the selected circuit parameters fitted to the impedance curve for $18650 \mathrm{LiFePO}_{4}$ battery cell under over-charge and over-discharge conditions, respectively. Where, circuit parameter $R_{0}$ is same as the bulk electrolyte resistance, $R_{b}$. Interested readers are encouraged to read more information on EIS from [푸 $\underline{18}, \underline{26}, \underline{27}]$.

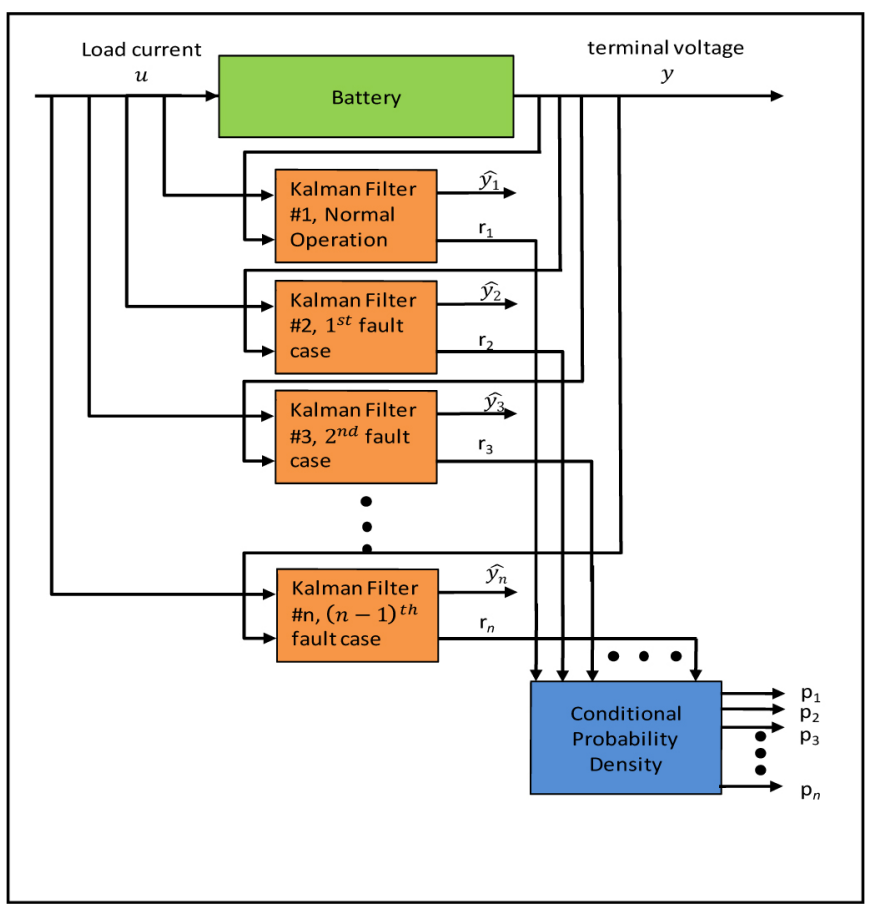

Fig. 3. Multiple-model residual generation and probability evaluation.

Table I. Impedance spectroscopy data in nominal discharge/ over-charge

\begin{tabular}{|l|l|l|l|l|l|}
\hline \multicolumn{7}{|c|}{ Overcharge } \\
\hline Cycle & $\mathbf{R}_{\mathbf{0}}(\mathbf{\Omega})$ & $\mathbf{C}(\mathbf{F})$ & $\mathbf{R}(\boldsymbol{\Omega})$ & $\mathbf{C}_{\text {dl }}(\mathbf{F})$ & $\mathbf{R}_{\text {ct }}(\mathbf{\Omega})$ \\
\hline $\mathbf{1}$ & 0.0771 & 0.0265 & 0.0156 & 0.4177 & 0.0282 \\
\hline $\mathbf{5}$ & 0.2433 & 0.0041 & 0.0369 & 0.2463 & 0.0329 \\
\hline $\mathbf{1 0}$ & 0.1395 & 0.0018 & 0.0720 & 0.1651 & 0.0376 \\
\hline $\mathbf{1 2}$ & 0.1387 & 0.0012 & 0.1429 & 0.1007 & 0.0500 \\
\hline $\mathbf{1 5}$ & 0.2865 & 0.0010 & 0.2571 & 0.0589 & 0.0763 \\
\hline $\mathbf{1 8}$ & 0.1661 & 0.0007 & 0.4907 & 0.0140 & 0.1833 \\
\hline
\end{tabular}

Table II. Impedance spectroscopy data in nominal charge/ over discharge

\begin{tabular}{|l|l|l|l|l|l|}
\hline \multicolumn{7}{|c|}{ Over-Discharge } \\
\hline Cycle & $\mathbf{R}_{\mathbf{0}}(\mathbf{\Omega})$ & $\mathbf{C}(\mathbf{F})$ & $\mathbf{R}(\mathbf{\Omega})$ & $\mathbf{C}_{\text {dd }}(\mathbf{F})$ & $\mathbf{R}_{\mathbf{c t}}(\mathbf{\Omega})$ \\
\hline $\mathbf{1}$ & 0.0503 & 0.1922 & 0.0051 & 0.8213 & 0.0126 \\
\hline $\mathbf{2}$ & 0.0566 & 0.2623 & 0.0045 & 2.6470 & 0.0098 \\
\hline $\mathbf{3}$ & 0.0578 & 0.2669 & 0.0055 & 3.2500 & 0.0123 \\
\hline $\mathbf{4}$ & 0.0594 & 0.4379 & 0.0053 & 4.2580 & 0.0126 \\
\hline $\mathbf{5}$ & 0.0569 & 0.4067 & 0.0056 & 4.3360 & 0.0112 \\
\hline $\mathbf{6}$ & 0.0623 & 0.2590 & 0.0054 & 2.9430 & 0.0081 \\
\hline
\end{tabular}

To mimic the actual operation, the battery is excited using the urban dynamometer driving schedule (UDDS) based current profile for an electric vehicle which has been scaled appropriately to match the nominal capacity of one battery cell. The UDDS profile is obtained using Autonomie [28]. The load current profile is as shown in Figure 4. The duration of cycle considered for the study is 71 seconds.

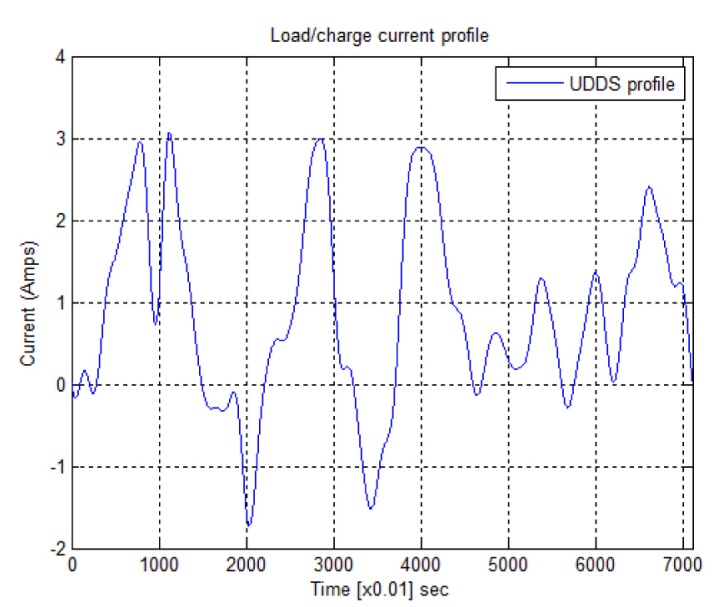

Fig. 4. Battery cell UDDS current profile.

At each sample, all the models get access to the same load/ charge current and terminal voltage measurements. Based on these inputs to Kalman filters or the extended Kalman filters, the estimated terminal voltage are generated. Further these terminal voltages are used in residual generation and evaluation. The corresponding simulated terminal voltage profile is as shown in Fig 5 .

For effective fault detection, the SOC is lower and upper bound to zero and $100 \%$ respectively. This measure ensures that the OCV remains under permissible limits, thus weighing the polarization voltages more [29]. The polarization voltages are the voltage drops across the constant phase element, $\mathrm{C}$, and the double layer capacitance, $C_{d l}$, elements in the equivalent circuit. The initial state of the system is considered to be $\left[\begin{array}{lll}0.7 & 0 & 0\end{array}\right]^{T}$ which implies $70 \%$ SOC and zero polarization voltages.

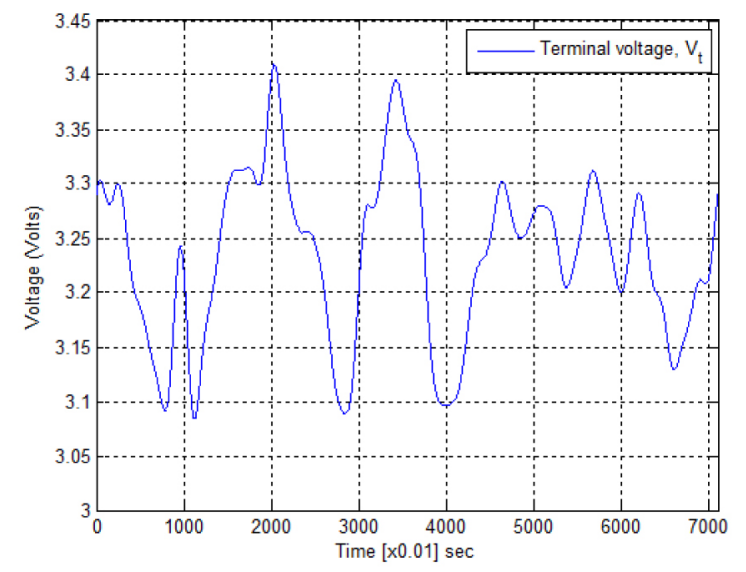

Fig. 5. Battery cell UDDS terminal voltage response.

\section{Diagnosis Performance Evaluation}

The fault diagnosis technique is introduced in $[\underline{8}, \underline{29}]$. To simulate the degree of effectiveness introduced by the linear and non-linear battery model in accurate fault detection and diagnosis, parameter 
variation is induced in the terminal voltage measurement to represent the consecutive fault cases. Based on the general trend of parameter variation observed during IS along with the degree of fault to be studied, the parameter values for over-charge and over-discharge conditions are selected. These conditions are further used to generate the fault carrying terminal voltage measurements. The total simulation time of 71 seconds is divided into four equal parts which occur consecutively.

1. Healthy battery condition: zero to 17.75 seconds.

2. Overcharge battery condition: 17.76 to 35.5 seconds.

3. Over-discharge battery condition: 35.51 to 53.25 seconds.

4. Healthy battery condition: 53.26 to 71 seconds.

Reverting back to healthy battery condition in the end helps to check the effectiveness of the algorithm to de-latch itself from a diagnosed fault case [14]. The covariance values for system and measurement noise are $Q=7 \times 10^{-7}, R=1 \times 10^{-6}$. It is also assumed that only one type of fault can occur in the system at a given point in time.

\section{Linear Battery Model}

The terminal voltages for the measured, healthy, overcharge and over discharged conditions are as shown in Figure 6. The resulting residuals for the healthy, overcharge and over discharged conditions are as shown in Figure 7. As discussed earlier, the residuals are the direct result of comparing the estimated terminal voltages for each system with the measured terminal voltage.

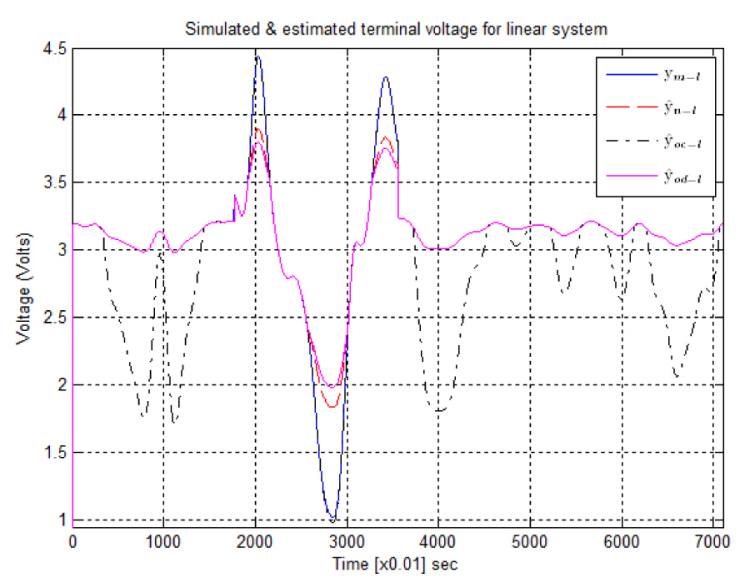

Fig 6. Terminal voltage for linear system models.

Due to the modeling inaccuracies caused by the assuming a linear OCV-SOC relationship, the residuals do not behave as expected.

Once these residuals are evaluated in the condition probability density block, the resulting system probabilities are as shown in the Figure 8.

Initially, during the first 10 seconds, the fault detection results are as expected. The system health probability $P_{H L}$ slowly starts to transition from 1 towards zero, indicating the departure from healthy battery operation. At the same time, the over discharge probability transitions from zero towards 1 . This behavior is unexpected and indicates inaccurate residual signals. From 17.76 to 35.5 seconds, the overcharge residuals becomes zero mean, and as a result, the fault probabilities transition to correct values and over-charge fault is indicated. At 35.51 seconds, the over-charge probability, as expected according to the evaluation scenario, transitions from zero to 1 , indicating the presence of over discharge fault condition. The fault detection setup then latches on to the over discharge fault condition, and fails to acknowledge the performance change at 53.26 seconds, to healthy battery operation.

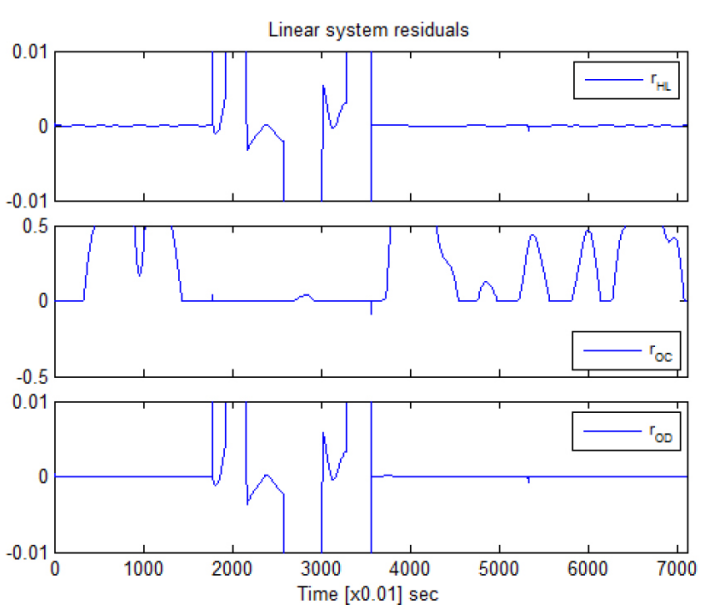

Fig. 7. Residuals for linear system models.

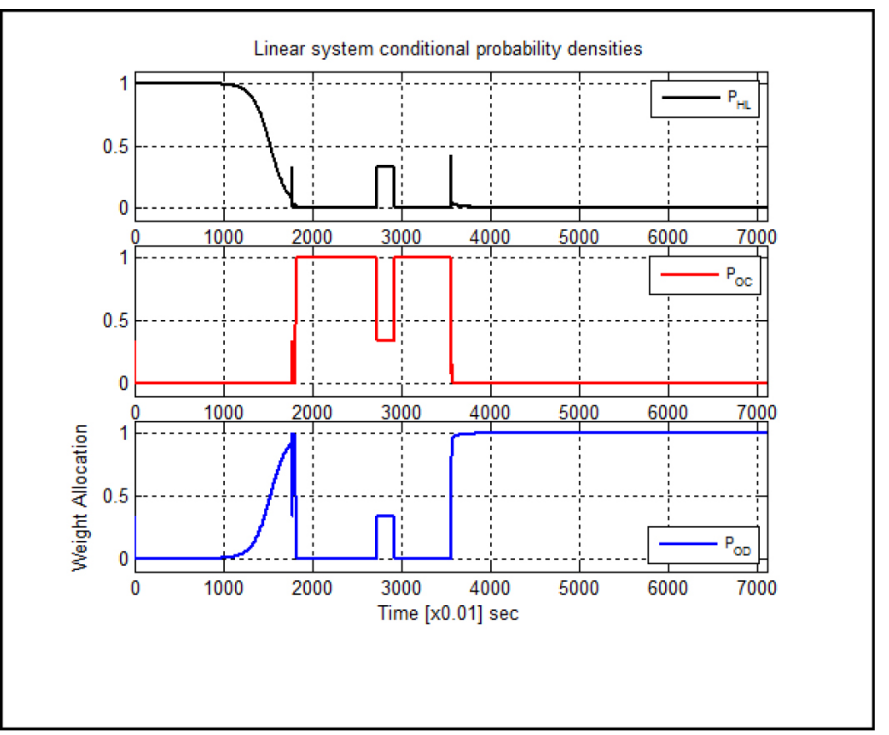

Fig. 8. Conditional probability densities for linear system.

\section{Non-Linear Battery Model}

With the non-linear system model, terminal voltage and hence the residuals carry more accurate information and indicate the expected fashion, as shown in Figures 9 and 10.

For the first and last 17.75 seconds of fault scenario, the healthy battery residual $r_{H L}$ shows a zero average value. From 17.76 to 35.5 seconds and later from 35.51 to 53.25 seconds, the over-charge residual $r_{O C}$ and over discharge residual $r_{O D}$ shows zero average signal behavior respectively. 


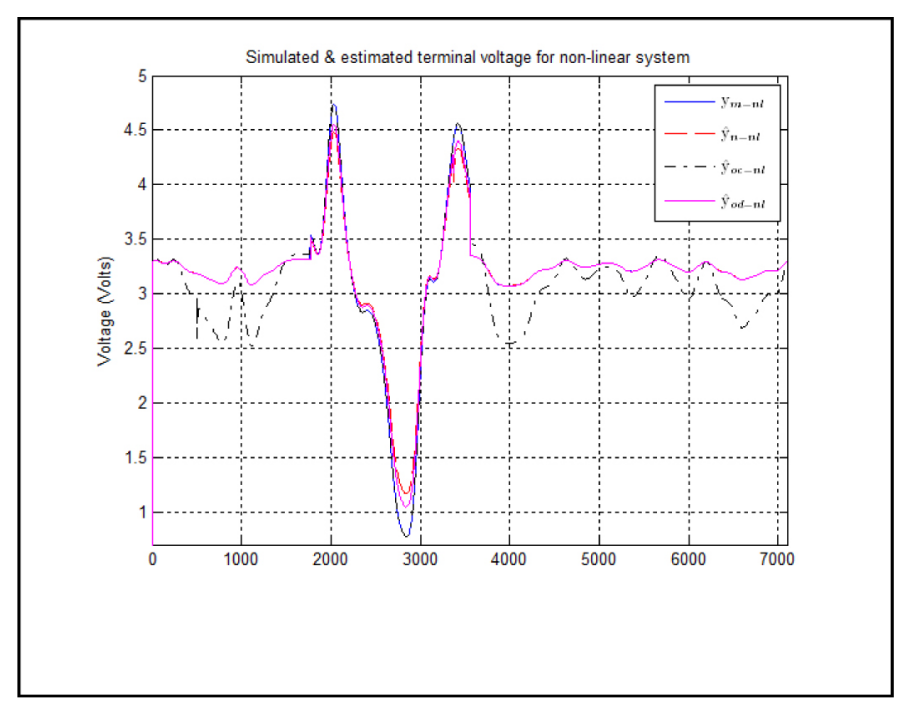

Fig. 9. Terminal voltage for non-linear system models.

Due to better approximation of the battery behavior by the non-linear models, the resulting estimated terminal voltages match closely the simulated terminal voltage measurement. This along with better noise approximation, results in minimal fault information loss and hence accurate residuals. The resulting conditional probability densities are as shown in Figure 11. The overcharge fault was injected at 17.75 seconds, which results in the healthy battery operation probability $P_{H L}$, reached zero, indicating the presence of a fault or the nonexistence of a healthy condition. The fault type was indicated by the probability $P_{O C}$ when it transitioned from 0 to 1 , indicating an over-charge fault. At the 35.5 seconds, probability $P_{O D}$ transitions to 1, while $P_{O C}$ reached zero, representing an over-discharge fault. The healthy cell operation was indicated at 53.25 seconds, when probability $P_{H L}$ transitioned to 1 and both $P_{O C}$ and $P_{O D}$ reached 0 .

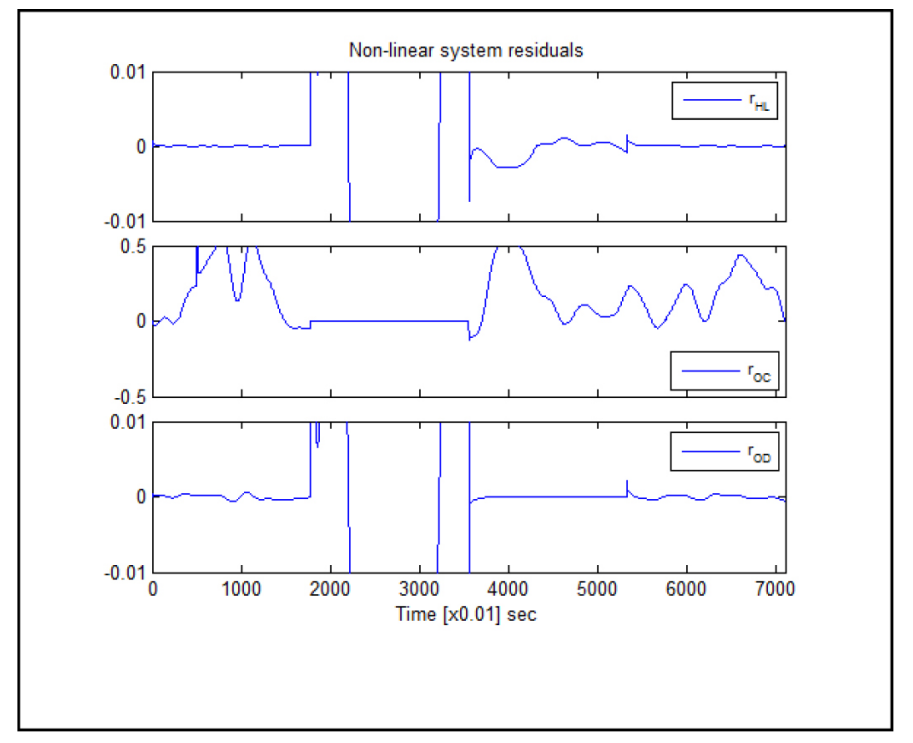

Fig. 10. Residuals for non-linear system models.

Accurate estimation of system and measurement noise variances has proven to profoundly impact on the residual generation process. Noise over- or under-estimation can result in loss of information during residual generation process, and hence affect the fault diagnosis performance [11]. The fault diagnosis performance as a function of battery model and the noise estimation is as shown in the surface plot of Figure 10. R and Q are the system and measurement noise variance, and $p p d$ stands for percentage positive detection. Within the performance evaluation scenario, $100 \%$ ppd indicates perfect fault detection while $0 \%$ ppd implies failure to detect the designed fault conditions. It is difficult to achieve $100 \%$ ppd because of the time it takes for the probabilities to transition from one extreme state to another, but a higher ppd indicates good fault detection and diagnosis performance. From Figure 12, it is clear that the nonlinear battery model, at 80 to $85 \mathrm{ppd}$, resulted in better fault detection and diagnosis when compared to its linear counterpart at 55 to $60 \mathrm{ppd}$. This difference can be attributed to better approximation of the battery dynamics offered by the non-linear battery model, which further leads to accurate estimation of the battery terminal voltage and eventually result in more accurate information in the residual generation process.

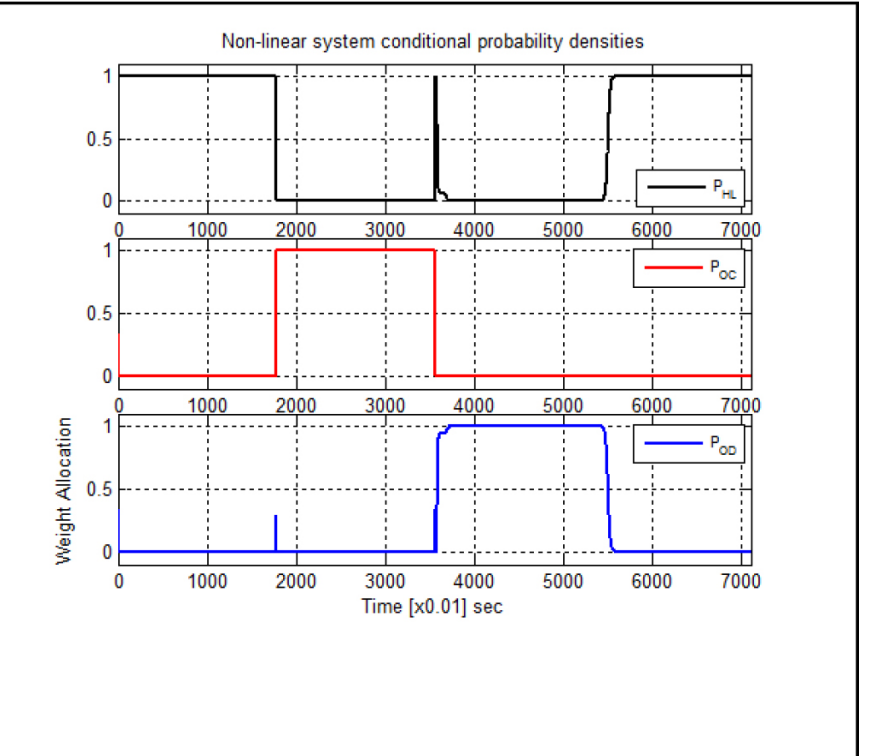

Fig. 11. Conditional probability densities for non-linear system.

The effect of model accuracy in fault detection can be analyzed from the performance evaluations presented in Figures $6-\underline{9}$. From time zero to 17.75 seconds, residual $r_{H L}$, for both the linear and non-linear models generated a zero average signal. The behavior of the system health residual in both the linear and non-linear model case is the direct result of the terminal voltage estimation for system health showing good agreement with the reference terminal voltage signal. In the same time frame, the residual $r_{O C}$ for both linear and non-linear models, demonstrated a largely expected deviation mainly because of the quality of over-charge terminal voltage estimation.

In overcharge, both models demonstrate a mismatch of probabilities during the first scenario. However, in the case of over-discharge, the fault residual $r_{O C}$ for the linear battery model showed a zero average behavior, which indicated that the terminal voltage estimate for over-discharge fault hypothesis in linear model was closer to the reference terminal voltage signal. As a result, a confusion was observed in the fault detection, and $P_{O C}$ incorrectly transitions from zero to 1 . At the same time, the non-linear model did perform better at capturing the fault dynamics and generated a dynamic overdischarge residual which captured the non-existence of overdischarge fault. Due to greater degree of parameter shift in overcharge condition, this fault was timely detected with both the linear and non-linear model case in the time range from 17.76 to 35.5 
seconds. A brief but critical loss in $P_{O C}$ and other inherently related probabilities in the linear case, was observed from 27.2 to 29.2 seconds where the $r_{O C}$ deviates from zero average behavior.

In the over-discharge region from 35.51 to 53.25 seconds, the residuals $r_{O D}$ and $r_{H L}$ for the linear battery model case showed a zero average behavior while $r_{O C}$ demonstrated some variations. In the non-linear model, the residual $r_{O D}$ became zero average while $r_{H L}$ and $r_{O C}$ showed dynamic behavior. This was indication of the fault insensitivity that could be caused by model uncertainty introduced by the linear battery model. Finally, from 53.26 to 71 seconds, for the non-linear model case, $r_{H L}$ became a zero average signal while $r_{O C}$ and $r_{O D}$ demonstrated dynamic behavior which was dependent on the difference between the estimated terminal voltages and the reference terminal voltage signal. This residual behavior led to effective fault de-latching as seen in the non-linear model probabilities. For the linear model, the faulty residuals $r_{H L}$ and $r_{O D}$ introduced ineffective fault diagnosis and hence led to the over-discharge fault probability latching.

For both linear and non-linear battery models, the overestimation of the process noise adversely affected the fault detection and diagnosis process and led to almost 15 to $20 \%$ drop in fault diagnosis performance, as shown in Figure 12. For a given magnitude of process noise, under-estimation of measurement noise also affected the fault diagnosis performance, hence leading to lower values of $p p d$. A combination of inaccurately estimated process and measurement noise as a whole resulted in loss of critical fault related information and further led to failure of the model-based fault detection setup.

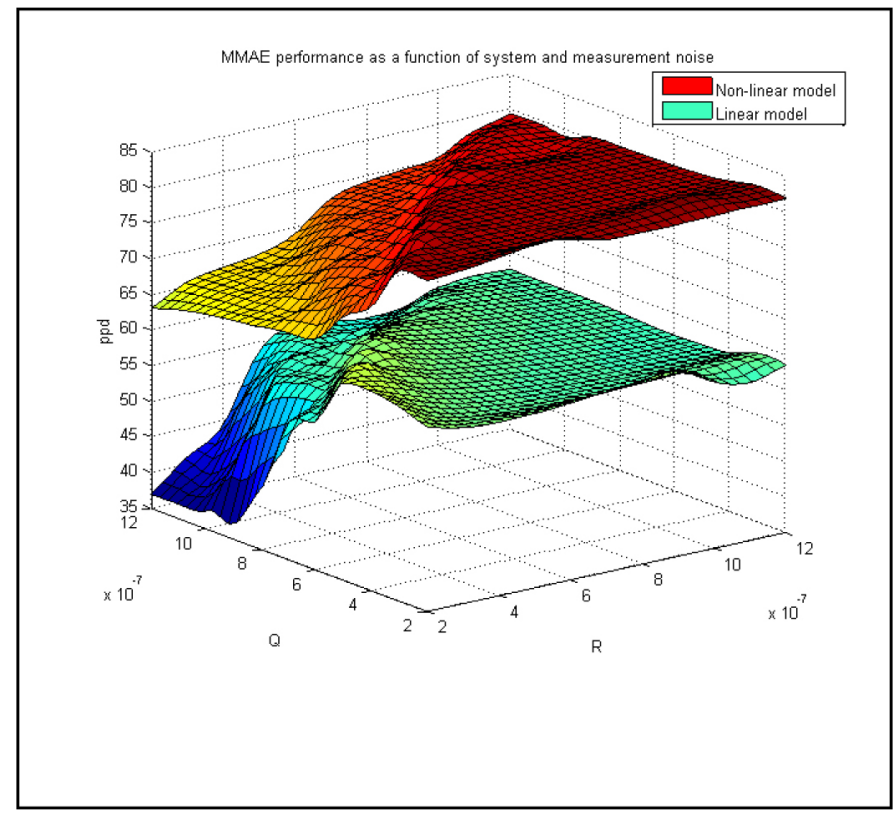

Fig. 12. Linear and non-linear battery model performance in fault diagnosis.

\section{Conclusion}

This paper investigated model based fault diagnosis using MMAE on li-ion battery for over-charge and over-discharge fault detection. Two different approaches to modeling the li-ion battery were considered and their effects on the diagnosis performance were presented. The non-linear battery model performed much better when it came to accurate and timely fault detection using multiple model adaptive estimation. The effects of noise over- and under-estimation were also considered, and their influence on fault decisions was studied. The robustness of fault detection technique dropped for both the linear and non-linear battery model based methods when the process and measurement noise was over- or under-estimated. However, it was shown that by reducing the model uncertainties and noise over- and underestimations, better fault diagnosis can be achieved for liion batteries. The effect of cell-to-cell variation and aging on the robustness of detection method, although not a part of this study, is currently under investigation by the authors.

\section{References}

1. Ding S. X., Model-based fault diagnosis techniques vol. 2013:Springer, 2008.

2. Isermann, R.Fault-diagnosis applications: model-based condition monitoring: actuators, drives, machinery, plants, sensors, and fault-tolerant systems: Springer, 2011.

3. Isermann R., Fault-diagnosis systems:Springer, 2006.

4. Reddy T. B., Linden's Handbook of Batteries vol. 4:McGrawHill, 2011.

5. Alavi S. M., Samadi M. F., and Saif M., "Diagnostics in Lithium-Ion Batteries: Challenging Issues and Recent Achievements," in Integration of Practice-Oriented Knowledge Technology: Trends and Prospectives, ed:Springer, 2013, pp. 277-291.

6. Chen W., Chen W. T., Saif M., Li M. F., and Wu H., "Simultaneous Fault Isolation and Estimation of Lithium-Ion Batteries via Synthesized Design of Luenberger and Learning Observers," Control Systems Technology, IEEE Transactions on, vol. PP, pp. 1-1, 2013.

7. Singh A., Izadian A., and Anwar S., "Fault Diagnosis of LiIon Batteries Using Multiple-Model Adaptive Estimation," presented at the IEEE Industrial Electronics, IECON 2013 39th Annual Conference on, Vienna, Austria, 2013.

8. Singh A., Izadian A., and Anwar S., "Model Based Condition Monitoring in Lithium-Ion Batteries," Journal of Power Sources, Vol 268, 2014, pp 459-468.

9. Maybeck P. S. and Pogoda D. L., "Multiple model adaptive controller for the stol $\mathrm{f}-15$ with sensor/actuator failures," in Decision and Control, 1989., Proceedings of the 28th IEEE Conference on, 1989, pp. 1566-1572.

10. Hanlon P. D. and Maybeck P. S., "Multiple-model adaptive estimation using a residual correlation Kalman filter bank," Aerospace and Electronic Systems, IEEE Transactions on, vol. 36, pp. 393-406, 2000.

11. Izadian A., "Self-Tuning Fault Diagnosis of MEMS," International Federation on Automatic Control, Journal of Mechatronics, Volume 23, Issue 8, December 2013, Pages 1094-1099.

12. Izadian A., Khayyer P., and Famouri P., "Fault Diagnosis of Time-Varying Parameter Systems With Application in MEMS LCRs," Industrial Electronics, IEEE Transactions on, vol. 56, pp. 973-978, 2009. 
13. Eide P. and Maybeck P., "An MMAE failure detection system for the F-16," Aerospace and Electronic Systems, IEEE Transactions on, vol. 32, pp. 1125-1136, 1996.

14. Izadian A. and Famouri P., "Fault Diagnosis of MEMS Lateral Comb Resonators Using Multiple-Model Adaptive Estimators," Control Systems Technology, IEEE Transactions on, vol. 18, pp. 1233-1240, 2010.

15. Xidong T., Xiaofeng M., Jian L., and Koch B., "Liion battery parameter estimation for state of charge," in American Control Conference (ACC), 2011, 2011, pp. 941-946.

16. Ranjbar A. H., Banaei A., Khoobroo A., and Fahimi B., "Online Estimation of State of Charge in Li-Ion Batteries Using Impulse Response Concept," Smart Grid, IEEE Transactions on, vol. 3, pp. 360-367, 2012.

17. Orazem M. E. and Tribollet B., Electrochemical Impedance Spectroscopy:John Wiley \& Sons, Inc., 2008.

18. Barsoukov E. and Macdonald J. R., Impedance Spectroscopy: Theory, Experiment, and Applications:Wiley, 2005.

19. Fei Z., Guangjun L., and Lijin F., "A battery State of Charge estimation method with extended Kalman filter," in Advanced Intelligent Mechatronics, 2008. AIM 2008. IEEE/ASME International Conference on, 2008, pp. 1008-1013.

20. Ogata K., Discrete-time control systems. Englewood Cliffs, N.J.: Prentice Hall, 1995.

21. Ross S. M., A first course in probability: Pearson Prentice Hall, 2010.

22. Abu-Sharkh S. and Doerffel D., "Rapid test and non-linear model characterisation of solid-state lithium-ion batteries," Journal of Power Sources, vol. 130, pp. 266-274, 2004.

23. Belov D. and Yang M.-H., "Failure mechanism of Li-ion battery at overcharge conditions," Journal of Solid State Electrochemistry, vol. 12, pp. 885-894, 2008/08/01 2008.

24. Mikolajczak C., Kahn M., White K., and Long R. T., "LithiumIon Batteries Hazard and Use Assessment," Fire Protection Research Foundation, 1 Batterymarch Park, Quincy, MA 02169-7471, ReportJuly 2011.

25. A. Systems. (2009, 9/9/12). High Power Li-Ion APR18650 [Data Sheet]. Available: www.cosmoenergy.com/APR18650M1A Datasheet 2009.pdf
26. Buller S., Thele M., De Doncker R. W. A. A., and Karden E., "Impedance-based simulation models of supercapacitors and Li-ion batteries for power electronic applications," Industry Applications, IEEE Transactions on, vol. 41, pp. 742-747, 2005.

27. Buller S., "Impedance-Based Simulation Models for Energy Storage Devices in Advanced Automotive Power Systems," Phd, Institute for Power Electronics and Electrical Drives, RWTH Aachen University, Germany, 2003.

28. laboratory A. N., "Autonomie," ed: Argonne National laboratory, 2010.

29. Singh A., Izadian A., and Anwar S., "Adaptive Nonlinear Model-Based Fault Diagnosis of Li-ion Batteries," IEEE Transactions on Industrial Electronics, Vol 62, No. 2, February, 2015, pp. 1002-1011.

\section{Contact}

Sohel Anwar is the corresponding author of this paper. He is currently an Associate Professor and Graduate Chair in the Department of Mechanical Engineering at Purdue School of Engineering and Technology, IUPUI, Indianapolis, IN 46202. He received his Ph.D. in Mechanical Engineering in 1995 from the University of Arizona, Tucson, AZ. Dr. Anwar worked for Caterpillar, Inc. from 1995 to 1999 where he was involved in developing novel X-by-wire control algorithms for wheel loaders. In 1999, he joined Ford Motor Company / Visteon Corporation where he focused on developing advanced fault tolerant control algorithms for drive by wire systems. Dr. Anwar holds 14 US patents and several foreign patents. He has published over 110 papers in refereed journals and conference proceedings. His research interests include battery modeling and diagnostics, autonomous driving, sensor development among others. He may be reached at

soanwar@iupui.edu

http://et.engr.iupui.edu/ soanwar/

\section{Acknowledgments}

The authors would like to thank Dr. J. Xie and Dr. Yadong for 18650 $\mathrm{LiFePO} 4$ impedance spectroscopy data. 